\title{
Infrared optical coatings for the EarthCARE Multispectral Imager
}

\author{
Gary Hawkins, ${ }^{1, *}$ David Woods, ${ }^{2}$ Richard Sherwood, ${ }^{1}$ and Karim Djotni ${ }^{1}$ \\ ${ }^{1}$ The University of Reading, Infrared Multilayer Laboratory, School of Systems Engineering, \\ Whiteknights, Reading, Berkshire RG6 6AY, UK \\ ${ }^{2}$ Surrey Satellite Technology Ltd., Rayleigh House, Sevenoaks, Kent TN14 5LJ, UK \\ *Corresponding author: g.j.hawkins@ reading.ac.uk
}

Received 2 July 2014; revised 28 August 2014; accepted 8 September 2014; posted 9 September 2014 (Doc. ID 215184); published 14 October 2014

\begin{abstract}
The Earth Cloud, Aerosol and Radiation Explorer mission (EarthCARE) Multispectral Imager (MSI) is a radiometric instrument designed to provide the imaging of the atmospheric cloud cover and the cloud top surface temperature from a sun-synchronous low Earth orbit. The MSI forms part of a suite of four instruments destined to support the European Space Agency Living Planet mission on-board the EarthCARE satellite payload to be launched in 2016, whose synergy will be used to construct three-dimensional scenes, textures, and temperatures of atmospheric clouds and aerosols. The MSI instrument contains seven channels: four solar channels to measure visible and short-wave infrared wavelengths, and three channels to measure infrared thermal emission. In this paper, we describe the optical layout of the infrared instrument channels, thin-film multilayer designs, the coating deposition method, and the spectral system throughput for the bandpass interference filters, dichroic beam splitters, lenses, and mirror coatings to discriminate wavelengths at $8.8,10.8$, and $12.0 \mu \mathrm{m}$. The rationale for the selection of thin-film materials, spectral measurement technique, and environmental testing performance are also presented. (C) 2014 Optical Society of America
\end{abstract}

OCIS codes: (120.6085) Space instrumentation; (260.3060) Infrared; (120.2440) Filters; (310.0310)

Thin films; (350.2460) Filters, interference; (310.6188) Spectral properties.

http://dx.doi.org/10.1364/AO.53.006983

\section{Introduction}

The Earth Cloud, Aerosol and Radiation Explorer mission (EarthCARE) [1] is a spaceflight instrument approved for the third Earth Explorer Core Mission in the Earth Observation Envelope Programme to quantify aerosol-cloud-radiation interactions for climate and numerical weather forecasting models. This is a major mission led by the European Space Agency (ESA) with a heritage of weather and climate satellite-borne instruments [2] to cover primary research objectives set out in the Living Planet Programme. EarthCARE is a cooperative mission between the ESA who are responsible for the entire

$1559-128 \mathrm{X} / 14 / 306983-10 \$ 15.00 / 0$

(C) 2014 Optical Society of America system, including the EarthCARE spacecraft, the launcher, and the ground segment, and the Japan Aerospace Exploration Agency (JAXA) who will provide a cloud profiling radar (CPR). The EarthCARE mission will help to determine the Earth radiation budget by providing global observations of vertical cloud and aerosol profiles. The mission is centered on the synergetic use of the data provided by an instrument suite consisting of an atmospheric LIDar, a CPR, a multispectral imager (MSI), and a broadband radiometer (BBR). Surrey Satellite Technology Ltd. (SSTL) is responsible for the MSI [3], which is a fixed-viewing nadir instrument to provide complementary data in support of the other EarthCARE instruments for the determination of cloud type, texture, temperature, and cloud phase. The instrument operates in seven spectral bands from $650 \mathrm{~nm}$ to 
$12 \mu \mathrm{m}$, comprising four solar channels at visible $(0.67 \mu \mathrm{m})$, near-infrared $(0.865 \mu \mathrm{m})$, and short-wave (SW) infrared (1.65 and $2.21 \mu \mathrm{m})$ wavelengths, and three channels $(8.8,10.8$, and $12.0 \mu \mathrm{m})$ measuring the thermal emitted radiation from Earth. The broadband antireflection coatings for the lenses in the fore and rear focusing optics are designed and supplied by Reynard Corporation. The University of Reading was responsible for the spectral design, fabrication, and testing of the thermal infrared (TIR) optical coatings for the integrated bandpass filter, dichroic, and mirror focal plane assembly.

\section{MSI TIR Optical Layout}

The optical design layout of the TIR channels on the MSI instrument [4] is illustrated in Fig. 1. This design concept uses a single $384 \times 288$ pixel area amorphous silicon microbolometer detector system array [5]. Radiation from the atmospheric target is received through the Earth viewport and is reflected off the calibration mirror. The beam is focused with an imaging lens at the optical aperture, which is then reflected off a fixed fold mirror and through a second imaging lens into the assembly of dichroics, mirrors, and bandpass filters as shown in optical design layout schematics in Figs. $\underline{2}$ and $\underline{3}$.

The two dichroic beam splitters at a $30^{\circ}$ incident tilt split the incident beam into three components for the wave bands centered at 8.8, 10.8, and $12.0 \mu \mathrm{m}$. The three beams are then folded through the bandpass filters onto a common image plane. Chromatic dispersion between channels at the image plane is minimized by judicious selection of the substrate thickness of the bandpass filters. The primary images of the target are formed by the second lens, which acts partly as a field lens to ensure the beams are telecentric through the dichroics and filters, and partly as a low-convergent beam of the fore-optics to minimize the effects of the illuminating cone, with a maximum angle of $10^{\circ}(\cong f / 5$ numerical aperture). The subsequent rear optics are enclosed in a temperature-controlled cavity, through which the three

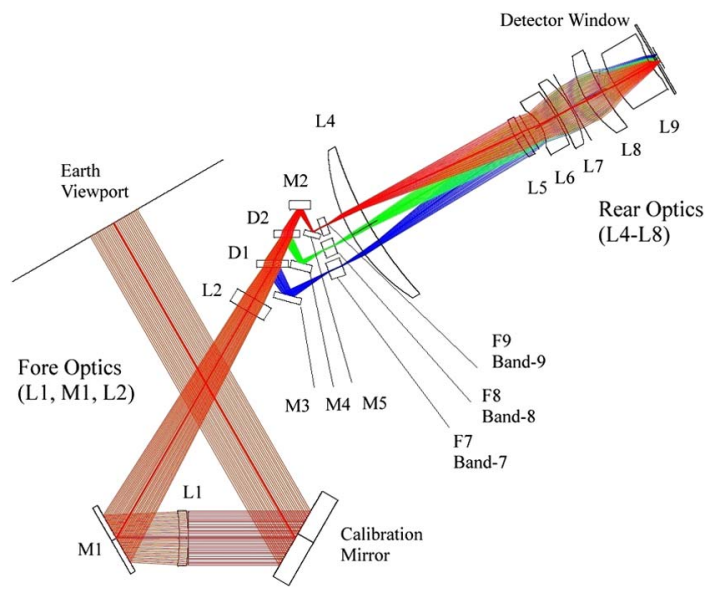

Fig. 1. MSI TIR optical layout.

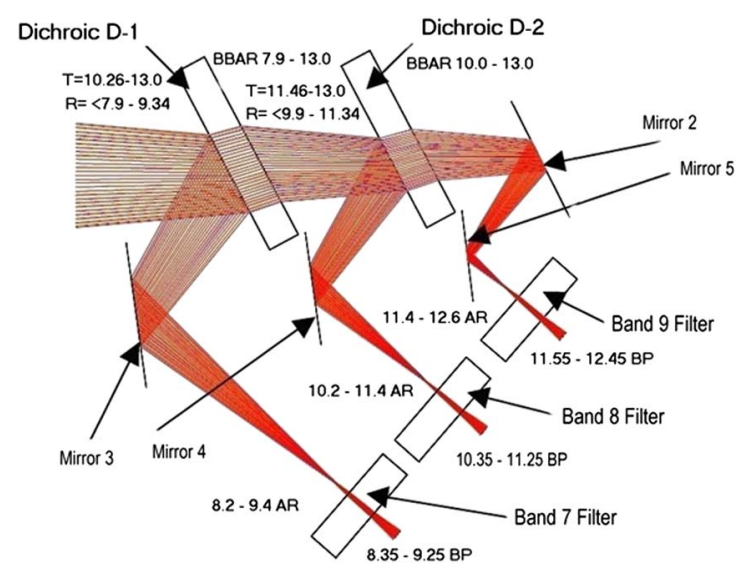

Fig. 2. MSI integrated bandpass filter, dichroic, and mirror assembly coating layout.

beams are re-imaged onto the detector with a maximum angle of $25^{\circ}(\cong f / 2$ aperture).

\section{Design Requirements}

As a driving priority of the MSI instrument is to achieve the highest quality of target image, the metrology and surface quality of substrate and coating materials were required to be precisely specified with high manufacturing tolerances. The dichroic, bandpass filter, and mirror substrates were all designed and manufactured as rectangular crystal prisms using optical-grade, low-inhomogeneity polished chemical vapor deposition (CVD) ZnSe material. The rectangular clear apertures required a typical surface flatness and parallelism of $<\lambda$ (incident surface) and $5 \lambda$ (rear surface) irregularity at the $\lambda=633 \mathrm{~nm}$ measurement wavelength, and $<2$ arcmin across the $120 \mathrm{~mm}$ long axis. Surface quality required a scratch-and-dig specification of 40-20 to military specification MIL-O-13830A and a surface roughness of $<10 \mathrm{~nm}$ RMS across the clear

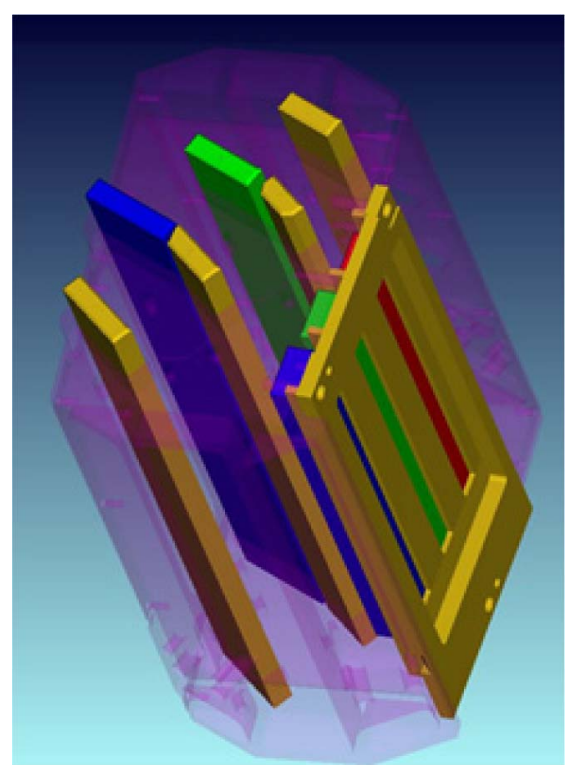

Fig. 3. Filter and dichroic design layout. 


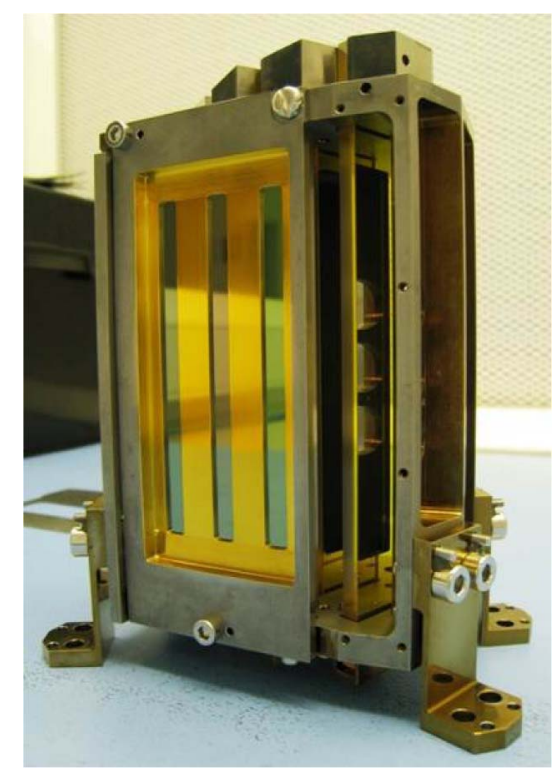

Fig. 4. Manufactured filter and dichroic optical assembly.

aperture. Focusing lenses further required a spherical surface departure of $<1$ fringe at $633 \mathrm{~nm}$ peakvalley (PV) over the clear aperture. The practical implementation of these requirements necessitated the use of substrate materials with limited opportunity for optical reworking. Hence, meticulous attention during each processing stage was crucial, requiring precision substrate fixtures, deposition plant engineering, high thermal contact conductance, and reproducible operating parameters to control the layer thickness accuracy at the elevated temperatures [6]. All substrate materials were manufactured and supplied by ULO Optics Ltd., UK. The manufactured engineering model of the filter and dichroic module assembly is shown in Fig. 4.

The spectral design of the instrument to discriminate the three thermal wave bands placed the specification requirements $[7,8]$ detailed in Tables 1 and 2 to define the passband properties and to achieve the desired end-to-end system throughput. The dichroic beam splitters designated as D1 and D2 with 50\% edge positions were located at wavelengths of 9.8 and $11.4 \mu \mathrm{m}$, respectively. The visible and nearinfrared channels were designated as Bands 1-4, at $0.67,0.865,1.65$, and $2.21 \mu \mathrm{m}$, and the TIR channels were named Bands 7-9, at 8.8, 10.8, and $12.0 \mu \mathrm{m}$. Bands 5 and 6 were discontinued at an early design phase of the instrument. The accuracy of the center wavelength placement required the center wavelength tolerance $\Delta \lambda / \lambda_{0}$ to be better than $0.6 \%$ across the operating range of the instrument.

Polarization sensitivity functions were calculated for design and measurements across the passbands at the operative angles of incidence using the definition of $\mathrm{DT}_{p-s}$ in Eq. (1), which describes the relative transmission ratio between the polarization difference and sum:

Table 1. Dichroic Beam Splitter Performance Requirements

\begin{tabular}{lcc}
\hline Requirement & Dichroic D1 & Dichroic D2 \\
\hline Substrate material & CVD ZnSe & CVD ZnSe \\
Function & Dichroic beam splitter & Dichroic beam splitter \\
Dimensions & $120.5 \mathrm{~mm} \times 18.0 \mathrm{~mm} \times 4.0 \mathrm{~mm}$ & $120.5 \mathrm{~mm} \times 14.85 \mathrm{~mm} \times 4.0 \mathrm{~mm}$ \\
Incident coating type & Long-wave-pass edge filter & Long-wave-pass edge filter \\
Rear coating type & $8-13 \mu \mathrm{m}$ antireflection & $10-13 \mu \mathrm{m}$ antireflection \\
Operating temperature & $300 \mathrm{~K}(-10 \mathrm{~K} /+20 \mathrm{~K})$ & $300 \mathrm{~K}(-10 \mathrm{~K} /+20 \mathrm{~K})$ \\
Angle of incidence & $30.0^{\circ}\left( \pm 6^{\circ}\right)$ & $30.0^{\circ}\left( \pm 6^{\circ}\right)$ \\
Reflection wave band & $<7.90-9.34 \mu \mathrm{m}$ & $<9.90-11.34 \mu \mathrm{m}$ \\
Transmission wave band & $10.26-13.0 \mu \mathrm{m}$ & $11.46-13.0 \mu \mathrm{m}$ \\
Antireflection wave band & $R \%<1 \%$ & $R \%<1 \%$ \\
Polarization sensitivity & $8.2-12.6 \mu \mathrm{m} \quad \mathrm{D}_{p-s}$ & $\mathrm{DT}_{p-s}(\lambda)$ and $\mathrm{DR}_{p-s}(\lambda) \leq 2 \%$ \\
\hline
\end{tabular}

Table 2. Bandpass Filter Performance Requirements

\begin{tabular}{lccc}
\hline Requirement & Bandpass F7 $(8.8 \mu \mathrm{m})$ & Bandpass F8 $(10.8 \mu \mathrm{m})$ & Bandpass F9 $(12.0 \mu \mathrm{m})$ \\
\hline Substrate material & CVD ZnSe & CVD ZnSe & CVD ZnSe \\
Function & Wide bandpass & Wide bandpass & Wide bandpass \\
Incident coating type & $8-10 \mu \mathrm{m}$ antireflection & $10-12 \mu \mathrm{m}$ antireflection & $11-13 \mu \mathrm{m}$ antireflection \\
Dimensions & $80.0 \mathrm{~mm} \times 10.1 \mathrm{~mm} \times 8.1 \mathrm{~mm}$ & $80.0 \mathrm{~mm} \times 9.8 \mathrm{~mm} \times 6.6 \mathrm{~mm}$ & $80.0 \mathrm{~mm} \times 10.1 \mathrm{~mm} \times 4.0 \mathrm{~mm}$ \\
Rear coating type & Long/short-wave pass & Long/short-wave pass & Long/short-wave pass \\
Operating temperature & $300 \mathrm{~K}(-10 \mathrm{~K} /+20 \mathrm{~K})$ & $300 \mathrm{~K}(-10 \mathrm{~K} /+20 \mathrm{~K})$ & $300 \mathrm{~K}(-10 \mathrm{~K} /+20 \mathrm{~K})$ \\
Angle of incidence & $9.5^{\circ}\left( \pm 6^{\circ}\right)$ & $9.5^{\circ}\left( \pm 6^{\circ}\right)$ & $9.5^{\circ}\left( \pm 6^{\circ}\right)$ \\
Transmission wave band & $8.35-9.25 \mu \mathrm{m}$ & $10.35-11.25 \mu \mathrm{m}$ & $11.55-12.45 \mu \mathrm{m}$ \\
Center wavelength & $8.80 \mu \mathrm{m}( \pm 0.05 \mu \mathrm{m})$ & $10.80 \mu \mathrm{m}( \pm 0.05 \mu \mathrm{m})$ & $12.0 \mu \mathrm{m}( \pm 0.05 \mu \mathrm{m})$ \\
Bandwidth/FWHM\%) & $0.9 \mu \mathrm{m} / 10.2 \%$ & $0.9 \mu \mathrm{m} / 8.3 \%$ & $0.9 \mu \mathrm{m} / 7.5 \%$ \\
$5 \%$ Bandwidth & $1.1 \mu \mathrm{m}$ & $1.1 \mu \mathrm{m}$ & $1.1 \mu \mathrm{m}$ \\
Antireflection band & $8-10 \mu \mathrm{m} R \%<1 \%$ & $10-12 \mu \mathrm{m} R \%<1 \%$ & $11-13 \mu \mathrm{m} R \%<1 \%$ \\
Polarization sensitivity & $\mathrm{DT}_{p-s}(\lambda)$ and $\mathrm{DR}_{p-s}(\lambda)<2 \%$ & $\mathrm{DT}_{p-s}(\lambda)$ and $\mathrm{DR}_{p-s}(\lambda)<2 \%$ & $\mathrm{DT}_{p-s}(\lambda)$ and $\mathrm{DR}_{p-s}(\lambda)<2 \%$ \\
\hline
\end{tabular}


Table 3. Bandpass Measurements of the Manufactured Flight Filters at Operating Parameters

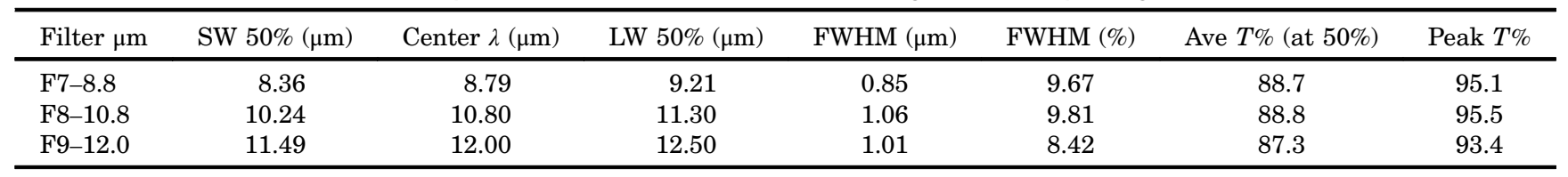

Table 4. Predicted End-To-End System Throughput from the Manufactured Flight Elements at their Operating Parameters

\begin{tabular}{lccccccc}
\hline Wave band \# $(\mu \mathrm{m})$ & SW 50\% $(\mu \mathrm{m})$ & Center $\lambda(\mu \mathrm{m})$ & LW 50\% $(\mu \mathrm{m})$ & FWHM $(\mu \mathrm{m})$ & FWHM $(\%)$ & Ave T\% $($ at 50\%) & Peak T\% \\
\hline $7-8.8$ & 8.36 & 8.79 & 9.22 & 0.86 & 9.78 & 72.5 & 79.3 \\
$8-10.8$ & 10.26 & 10.785 & 11.31 & 1.05 & 9.74 & 72.4 & 78.0 \\
$9-12.0$ & 11.52 & 12.00 & 12.48 & 0.96 & 8.00 & 50.2 & 59.7 \\
\hline
\end{tabular}

$\mathrm{DT}_{p-s}(\lambda)=\frac{T_{p}-T_{s}}{T_{p}+T_{s}}, \quad$ where $T_{p}=\int_{\lambda \min }^{\lambda \max } T_{p}(\lambda) \mathrm{d} \lambda$

and $\quad T_{s}=\int_{\lambda \min }^{\lambda \max } T_{s}(\lambda) \mathrm{d} \lambda$.

$T_{p}$ and $T_{s}$ are the linearly polarized transmission states exhibited at the $30^{\circ}$ incident angle of the dichroics, measured through a spectrometer polarizer at $0^{\circ}$ vertical polarization, $\left(S\right.$-pol), and $90^{\circ}$ horizontal polarization, $(P$-pol). The spectral requirement for the separation of polarization states required this summation to be less than $2 \%$ over the entire passband.

The ratio of in-band (IB) transmission to out-ofband (OOB) blocking is calculated with Eq. (2) for both design and measurements (with a $10^{-4}$ measurement sensitivity limit) across the full spectral width for each band:

$$
\begin{aligned}
\operatorname{OOB}(\lambda) & =1-\frac{\operatorname{InBand}(\mathrm{IB})}{\text { TotalBand(TB) }}, \\
\text { where TB } & =\int_{\lambda \min }^{\lambda \max } T \%(\lambda) \mathrm{d} \lambda \\
\text { and IB } & =\int_{\text {Sw0.1\% }}^{\mathrm{LW} 0.1 \%} T_{\mathrm{IB}} \%(\lambda) \mathrm{d} \lambda .
\end{aligned}
$$

Cut-off transition between passband and blocking was defined at $0.1 \%\left(10^{-3}\right)$ of the SW and long-wave (LW) band edges.

\section{Coating Designs}

The spectral design of the dichroics and bandpass filters shown in Figs. 5 and 6 principally comprised combinations of long-wave-pass and short-wave-pass edge filters. The dashed vertical lines represent the maximum spectral wavelength limits. Bandpass cuton and cut-off edges, together with the OOB blocking multilayers, were designed and matched optically as a combined thin-film structure, and were deposited in-series on the rear substrate surface. The multilayers contained various overlapping combinations of refined Tschebyscheff [9] polynomial designs and quarter-wave stacks, with appropriate indexmatching between stacks and antireflection layers.

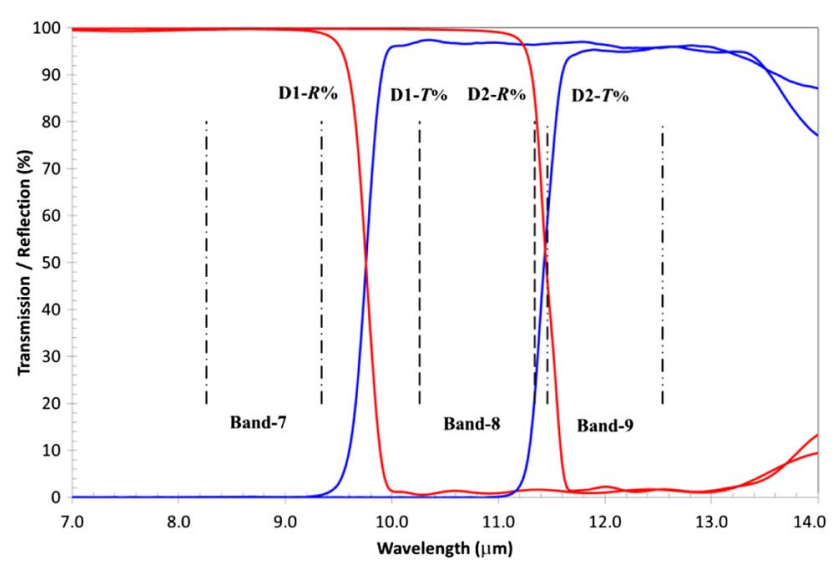

Fig. 5. Overlay of the manufactured dichroic measurements at $30^{\circ}$ incidence and spectral limits.

Broadband antireflection coatings were designed and deposited on the opposing surface of each optic to maximize the IB channel throughput. These antireflection coatings shown in Fig. 7 comprised multilayer designs with a range of bandwidth ratio between $\sim 1.2-1.6: 1$ across the differing channel wave bands.

The principal coating design for dichroic D1 spectrally comprised an optimized Tschebyscheff longwave-pass edge filter to reflect the wavelengths

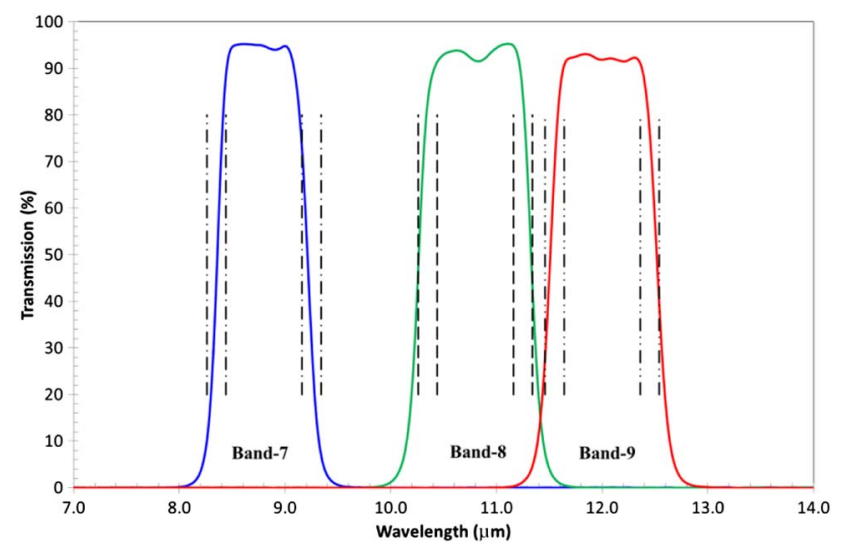

Fig. 6. Overlay of the measured bandpass filters at $295 \mathrm{~K}$ under $9.5^{\circ}$ incident illumination and spectral tolerances. 


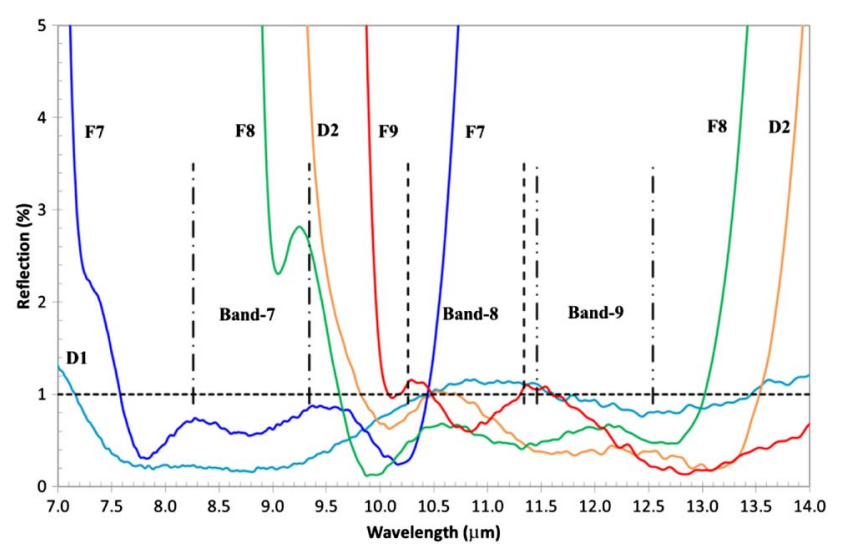

Fig. 7. Overlay of the manufactured broadband antireflection coatings $(R \%<1 \%)$.

across the 7.9-9.34 $\mu \mathrm{m}$ wave band and to transmit wavelengths between 10.26 and $13.0 \mu \mathrm{m}$. The multilayer deposited on the rear surface was a 13-layer optimized 7-14 $\mu \mathrm{m}$ broadband antireflection of alternate $\mathrm{PbTe} / \mathrm{ZnSe}$ composition. This multilayer was further overcoated with a low-index layer of $\mathrm{BaF}_{2}$, essential to maximize the performance throughout the antireflection zone. The close spectral proximity between Channel 8 and 9 wave bands required the refinement of a steep long-wave-pass edge filter $\left(\left(\lambda_{80 \% T}-\lambda_{5 \% T} / \lambda_{50 \% T}\right)=1.7 \%\right.$ at $0^{\circ}$ and $2.4 \%$ at $30^{\circ}$ incidence) to reflect the wavelengths across the 7.9-11.34 $\mu \mathrm{m}$ region, while transmitting wavelengths between 11.46 and $13.0 \mu \mathrm{m}$. Within practical design and manufacturing constraints, the diminishing increase of edge steepness with layer count inevitably results in an infringement between these wave bands, as shown with polarization in Fig. 8 . However, this encroachment was deemed acceptable as a consequence of thin-film interference limitations within the constrained refractive index contrast, and had a minimal influence on the total integrated spectral throughput budget. The rear surface comprised a 17-layer $\mathrm{PbTe}$ and $\mathrm{ZnSe}$ antireflection to maximize transparency across the $9.5-14.0 \mu \mathrm{m}$ region. Angular sensitivity $(d \lambda / d \theta)$ of the dichroic designs with polarization is shown in Fig. 9.

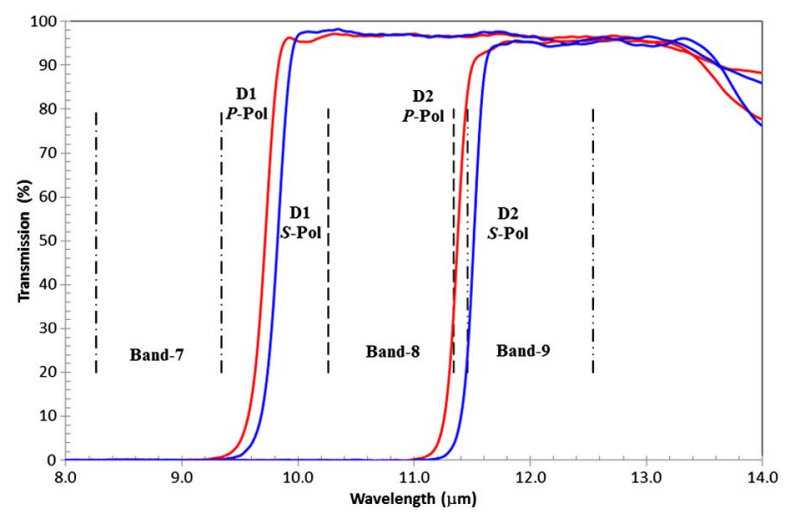

Fig. 8. Measurements of dichroic polarization split at $30^{\circ}$ incidence, with the polarizer at $0^{\circ}(S$-pol $)$ and $90^{\circ}(P$-pol $)$.

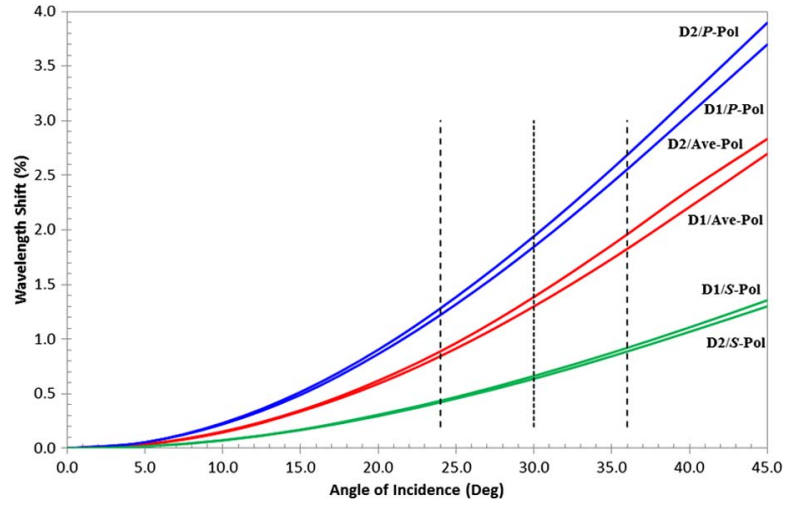

Fig. 9. Overlay of simulated dichroic angle sensitivity and incident angle tolerance.

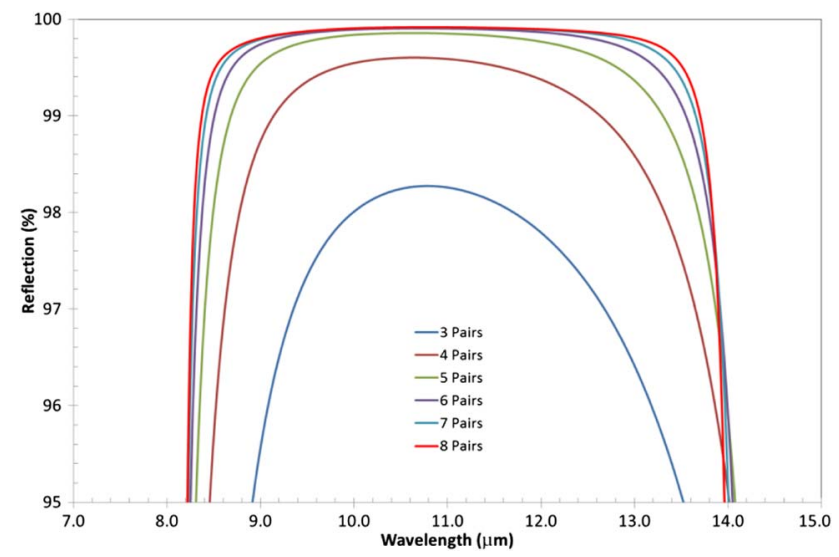

Fig. 10. Typical increasing bandwidth and reflectivity of a dielectric mirror with layer pair count.

The multilayer design of the dielectric mirrors comprised quarter-wave stacks of alternate $\mathrm{PbTe}$ $\left(n_{H}=5.5\right)$ and $\mathrm{ZnSe}\left(n_{L}=2.4\right)$ layer materials to provide high reflectance by in-phase constructive interference, and contained a low-index eighthwave outermost layer of ZnSe to aid optical impedance matching. As the rate of change in reflectivity diminishes with increasing layer count, as shown in Fig. 10, the multilayer mirror designs were constructed with a period of eight pairs to maximize both reflection intensity and bandwidth $(\Delta g=$ $2 / \pi \arcsin \left[n_{H}-n_{L} / n_{H}+n_{L}\right]$, where $\Delta g$ is half of the reflectance zone width).

\section{Infrared Material Properties}

The layout of the focusing optics for the MSI TIR wave bands [5] was particularly designed to accommodate the effect of temperature changes on the performance of the optical system. Several optical parameters can be affected by temperature variation, but the main challenge is to minimize focus shift and consequential degradation in image quality. The MSI was designed with athermalization corrections in mind to accommodate the effects of temperature on chromatic dispersion $(d \lambda / d T)$ and beam convergence. The athermal operation was achieved by using 


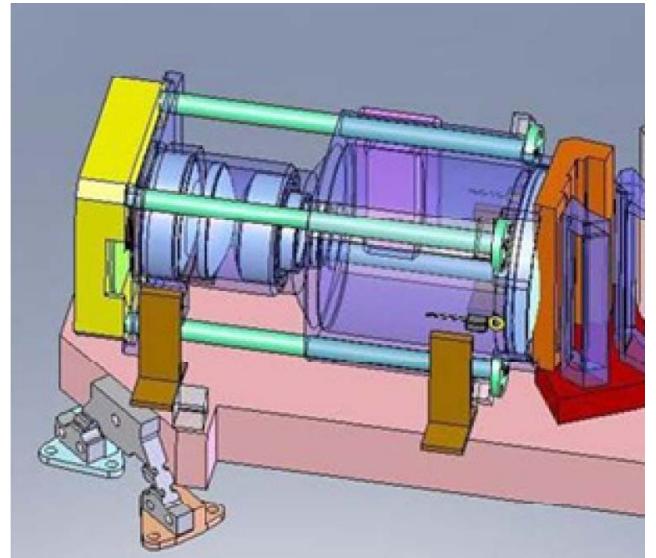

Fig. 11. Detector assembly with Invar rods.

Invar rods to connect to the detector assembly and was anchored to the front of the relay lens cell, as shown in Fig. 11. When the aluminum cell expands and contracts with temperature change $(d T)$, the detector remains stationary. This differential expansion corrects for the thermal focus drift over a wide temperature range $( \pm 60 \mathrm{~K})$, as shown in Fig. 12 for the geometric modulation transfer function (MTF) average at Nyquist frequency versus temperature.

Thermo-optical resilience was further achieved using a selection of differing lens materials to minimize refractive index changes that affect focusing shifts with temperature $(d n / d T)$, while maintaining high transparency across each wave band. The substrate materials comprised $\mathrm{ZnSe}, \mathrm{GaAs}$, and Ge with total integrated thicknesses of 64,16 , and $10 \mathrm{~mm}$, respectively, as shown in Fig. 13. ZnSe and GaAs exhibited high transparency throughout the selected wave bands; however, the commencement of intense LW multiphonon absorption in Ge at $11.5 \mu \mathrm{m}$ severely and inescapably attenuated the spectral throughput of the Channel 9 wave band at $12 \mu \mathrm{m}$. Additionally, KRS-5 thallium bromo-iodide (TlBr-TlI) was also considered for lens L3 in the optical train to assist with athermalisation, with its ability to maintain focus over a wide range of temperature and LW

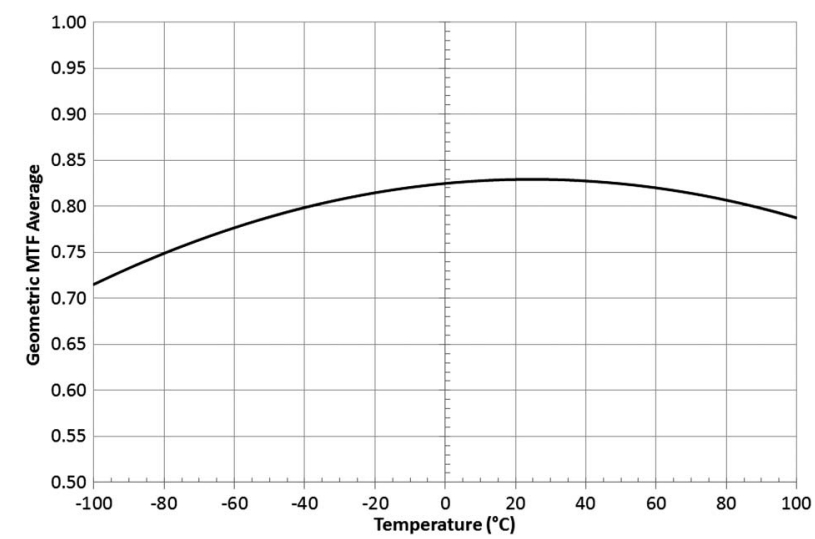

Fig. 12. Geometric MTF average at Nyquist frequency versus temperature.

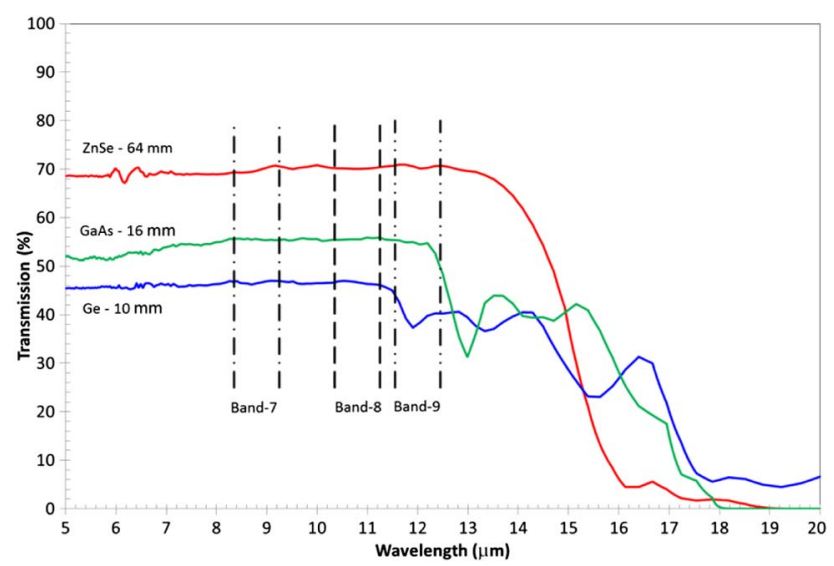

Fig. 13. Simulation of the total integrated transmission of uncoated $\mathrm{ZnSe}, \mathrm{Ge}$, and GaAs substrates at $295 \mathrm{~K}$.

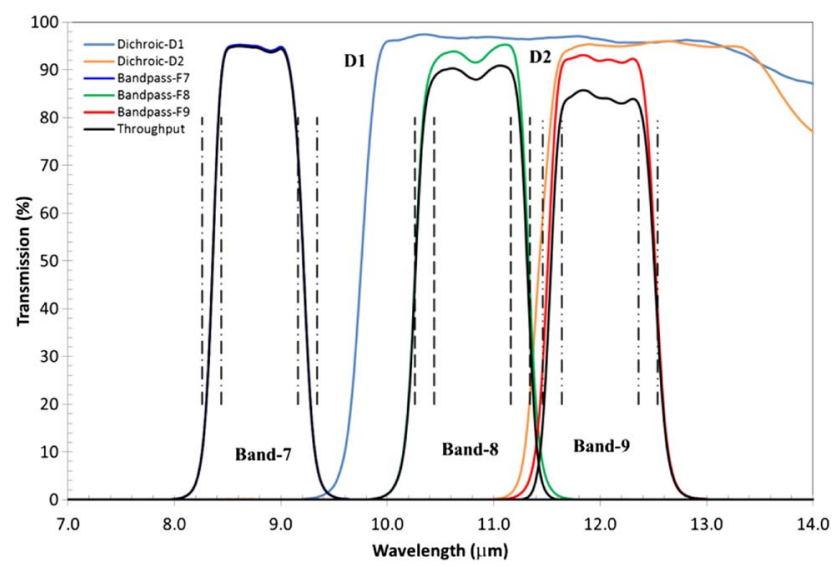

Fig. 14. Overlay of the transmission measurements of the dichroics and filters at their respective operating conditions, and the predicted throughput from the integrated assembly.

Reststrahl absorption maxima. This was, however, rejected at the time [10] due to high plastic deformation and internal strain [11] present in current KRS-5 crystal growth, precluding polishing to the standards necessary for high-quality imaging of these spaceflight optics.

$\mathrm{ZnSe}$ is a robust low-absorption substrate with high transparency across the $0.5-15 \mu \mathrm{m}$ range. It is essentially free of extrinsic impurity absorptions, providing extremely low bulk scatter losses. Having low absorption across wide interband transparency windows makes it very useful as the principal substrate material in the MSI multispectral optical design. The longer wavelength multiphonon absorption cutoff is due to a combination of weaker bonding heavier atoms and a more covalent bonding structure. This combination of weaker bonding and a high atomic weight generates lower frequency lattice vibrations [12]. The more covalent nature of these bonds results in less intense absorption through reduced dipole moment coupling. ZnSe is a cubic crystal manufactured by the CVD process with hydrogen selenide to produce superior optical properties to 
ZnS, often attributed to improved stoichiometry and the single cubic crystalline phase [13]. It can be polished optically to high flatness and parallel tolerance to provide good imaging characteristics for high resolution, and can correct for some color distortion inherent in the instrument. It is also capable of withstanding limited mechanical and cryogenic thermal shocks.

GaAs was particularly chosen as the desired material for inclusion in the MSI optical design for lenses L2 and L7. It possesses an intense multiphonon absorption feature commencing at $>18 \mu \mathrm{m}$, and is, therefore, capable of providing continuous rejection of long-wavelength radiation to suppress the microbolometer detector response across the unwanted far-infrared wavelengths. Ge was also included for lens L8 as it has the highest refractive index ( $n=4.0)$ of the infrared bulk transmitting substrate materials, and its low dispersion properties $\left(d n / d T=3.96 \times 10^{-4} / \mathrm{K}\right)$ across the temperature range avoids correction for chromatic aberration. This combination of high refractive index and low dispersion also aids imaging by minimizing the beam displacement of the incident illuminating cone. The intense multiphonon absorption in Ge that attenuates the $12 \mu \mathrm{m}$ wave band transparency is, however, unavoidable and complex, consisting of several types of phonon vibrations. These are primarily due to the transfer of energy from low-energy photons to free carriers, accompanied by the transfer of momentum between free carriers and phonons in the lattice [14].

The availability of transparent optical materials for thin-film interference filtering across the TIR spectral range is dominated by the chemistry of Group VI, II-VI, and IV-VI dielectric and semiconductor materials. With a high molecular mass, these materials transmit across a wide infrared interband region. The heaviest compound within this group is lead telluride ( $\mathrm{PbTe}$ ), which was used extensively as high-index $\mathrm{H}$-layers in the multilayer designs. PbTe as the high-index layer has been widely investigated over many years, principally due to its smallbandgap thermoelectric and semiconductor properties in optoelectronic devices [15], and provides various optical transparency and refractive index advantages compared with other Group IV coating materials [16]. The high refractive index [17] $(n=5.5$ at $\overline{295} \mathrm{~K})$ when used with an appropriate low-index II-VI dielectric results in a high index contrast to achieve the spectral filtering requirements with minimum number of layers and to maximize the spectral throughput. Zinc selenide (ZnSe, $n \sim 2.4$ ) was selected as the principal alternating low-index II-VI dielectric. This material provides a good index contrast with $\mathrm{PbTe}\left(n_{H} / n_{L}\right)$ and deposits as a highly durable hard coating with the same material properties as the underlying substrate, and has a heritage in many former spaceflight and astronomical instruments [18] for the mid-infrared wavelength region. At ambient room temperature, the bulk properties exhibit long-wavelength transparency up to $>15 \mu \mathrm{m}$, which as a thin film is further transparent up to around $25 \mu \mathrm{m}$.

\section{Thin-Film Fabrication}

The dichroic, mirror, and filter assembly coatings were deposited by thermal evaporation using a modified Balzers BA510 bell-jar evaporator. This deposition system is especially fitted with tooling for deposition of II-VI and IV-VI dielectric and chalcogenide materials with a configuration of rotating evaporation source geometry and stationary substrates, which has been reported extensively elsewhere $[19,20]$. This static substrate arrangement is especially important for the deposition of precision thin-film multilayers in the mid-infrared, particularly as the need for accurate elevated temperature control is essential to maintain good deposition uniformity and transparency, particularly as the sticking coefficient and stoichiometry of the materials are highly temperature-dependent [21].

The deposition thickness of each layer is monitored optically from an in situ reflected broadband infrared beam focused through a fixed-bandwidth monochromatic wavelength, selected at a high-order spectral position corresponding to the multilayer thickness range. The monochromator wavelengths and bandwidth are selected on proviso of maintaining signal strength and resolution, which may deteriorate from reflectance amplitude losses with accumulated multilayer thickness (viz., a narrow-bandwidth slit provides high resolution and reduces reflectance amplitude convergence with subsequent decrease of signal-to-noise ratio, whereas a wider slit grants higher signal throughput, but loss of resolution and increased amplitude convergence).

The infrared monitoring source was selected to be either a miniature $9 \mathrm{~W}$ infrared emitter operating at $\sim 1275^{\circ} \mathrm{C}$ for medium-wave infrared (MWIR) wavelengths greater than $4.5 \mu \mathrm{m}$ or a quartz halogen lamp for short-wave infrared wavelengths $(2.0-4.5 \mu \mathrm{m})$. A variable circular iris diaphragm positioned above the source as an aperture stop controlled the beam size incident on the optical monitor. The beam is chopped before entering the plant to negate any variable radiated flux originating from the evaporation sources and for suppression of electronic noise, using a multiblade rotating chopper. An optoelectronic photodiode switch measures the chopper position as a function of time to synchronize a reference signal relative to the detector response for the phase-sensitive detection system. Although this procedure provides an accurate method for controlled deposition of layers through accumulated multilayer thickness, the intrinsic accuracy is, however, still subject to both residual uncertainties of instrumental and operator errors. The fabrication of layers of non-quarter-wave thickness at the monitoring wavelength is achieved by the inclusion of a monitor shutter arrangement that isolates the filter substrates from the evaporant received by the optical monitor. The optical monitoring of the integral quarter-wave layers is terminated 
by a prescribed reflectance overshoot beyond the minimum to maintain amplitude and accuracy. Although this arrangement avoids the compounding of former thickness errors, is still subject to residual random errors affecting the precision of the spectral profile.

In addition to the sensitivity of optical thickness to deviations in monitoring during fabrication, the ultimate achievable spectral performance of the coating is also governed by limitations of design sensitivity to errors that may arise during the coating manufacture. As well as unidentified deviations in optical constant data, there are residual accuracy errors in the value of the actual deposited thickness of individual layers, and in the uniformity of thickness between substrates and across the surface area of the coating. By applying random errors to all of the layer thickness parameters simultaneously, the deviations indicate that a sensitivity of the intrinsic layer thickness accuracy better than $0.5 \%$ is required throughout the deposition to maintain spectral compliance.

\section{Spectral and Environmental Testing}

Spectral measurements for the MSI TIR coatings were performed using a Perkin-Elmer Spectrum 2000 "Optica" FTIR spectrophotometer. This instrument is especially adapted to address the ordinate accuracy concerns caused by inter-reflections (aliasing) and stray reflections from highly reflective optical samples reaching the detector together with detector nonlinearities and electronics [22]. The optical train of the spectrometer uses strategically placed half-apertures and optical masks. It forms a high mid-infrared energy grasp between 1.5 and $50 \mu \mathrm{m}$ using a deuterated triglycine sulphate (DTGS) detector and cesium iodide (CsI) beam splitter. The transmission measurements were performed in the sample compartment that contains a mechanical shuttle arrangement synchronized with the Fourier transform infrared (FTIR) operation to ratio between ambient background radiation and negate instrumental drift to provide a consistent measurement

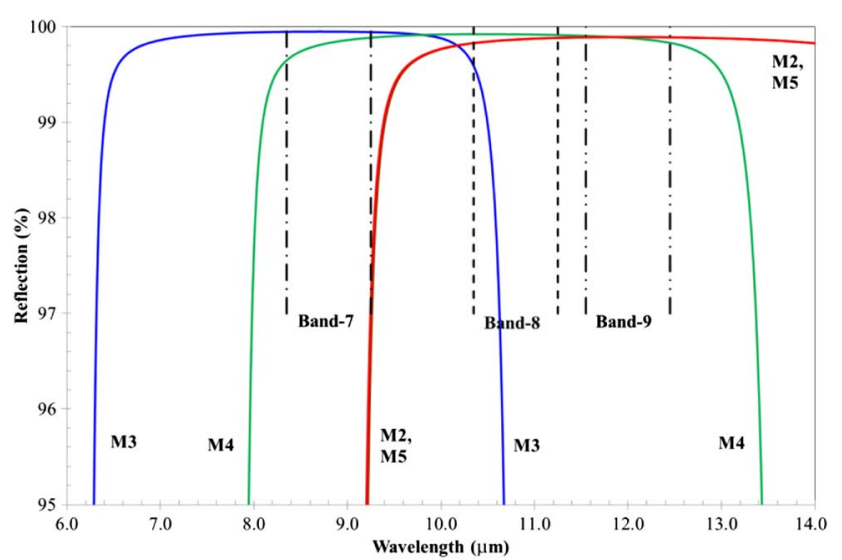

Fig. 15. Simulated performance of the manufactured dielectric mirrors at $45^{\circ}$ incidence. reference. Background and sample measurements were performed with a $7 \mathrm{~mm}$ diameter beam focus, and were alternated with a ratio of $1: 4: 1$. Measurements of polarization at $30^{\circ}$ incident angle for the dichroics and $9.5^{\circ}$ for the filters were performed using an internal mid-infrared polarizer positioned in the spectrometer optical beam and rotated at $0^{\circ}(S$-pol $)$ and $90^{\circ}(P$-pol). Transmission measurements of the discrete dichroic and filter elements, together with the predicted assembly throughput is shown in Fig. 14. Measured in-band spectral properties of manufactured flight model (FM) bandpass filters are shown in Table 3.

The spectral response in Fig. 15 shows the simulated reflectance performance predicted for the dielectric mirror coatings at $45^{\circ}$ incidence. The spectral reflectance measurements were performed on representative witness samples deposited in the same deposition using a spectrometer accessory designed with a fixed $16^{\circ}$ incident illumination angle. Simulations of wavelength positioning at $45^{\circ}$ were obtained by extrapolation using angle sensitivity from the thin-film multilayer design theory. Subsequent simulations of reflectance performance further used parameters determined from transmission measurements to infer the reflected performance obtained at normal incidence and at the $45^{\circ}$ incident angle. This included optical constant dispersion models of the complex refractive indices $(n=n-i k)$ of the substrate and layer materials.

In order to assess the environmental durability performance of the optical coatings, representative witness samples contained in the same deposition were subjected to the general provisions of the military specifications MIL-F-48616 and MIL-O-13830. These included visual assessment at $\times 20$ magnification, surface quality (40-5 scratch-dig), adhesion testing, moderate abrasion resistance, thermal shock testing $\left(77 \mathrm{~K}\right.$ to $+30^{\circ} \mathrm{C}$ ), and humidity testing at $40^{\circ} \mathrm{C}$ in $>95 \%$ relative humidity for $48 \mathrm{~h}$. Ionizing radiation required an accrued dose of $10 \mathrm{krad}(\mathrm{Si})$ with $3 \mathrm{~mm}$ shielding, as demonstrated by similarity [23]. This represented the equivalent average exposure received at the calibration mirror contributing a quarter of a steradian to open space.

\section{System Throughput Performance}

A system throughput model designed to simulate the spectral budget for each TIR channel was developed for the MSI to provide the science and engineering teams with a preview of predicted performance. This model uses both the calculated and measured spectral characteristics of each optical train element to determine the integrated end-to-end spectral throughput. The results of the model demonstrate the predicted instrument level performance, from which the mean IB throughput response is determined [24]. The predicted instrument throughput was derived by combining the spectral $R \%$ or $T \%$ properties of the components along the optical train, assuming absence of multiple beaming between 


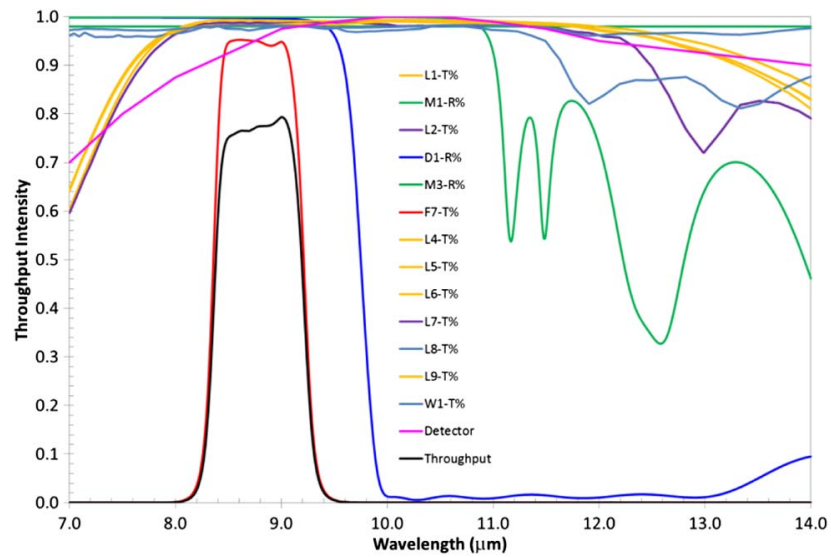

Fig. 16. Predicted total instrument throughput of Band 7 $(8.8 \mu \mathrm{m})$.

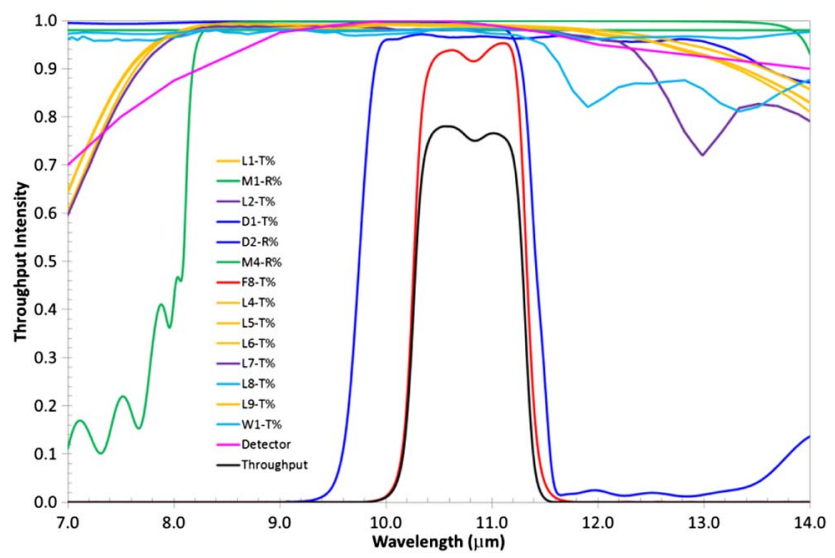

Fig. 17. Predicted total instrument throughput of Band 8 $(10.8 \mu \mathrm{m})$.

elements. The spectral profiles are suitably adjusted for their incident tilt angles and conical illumination. Using this method, the spectral measurements of the fabricated spaceflight elements, corrected for their appropriate angles, were substituted to verify the instrument performance. The predicted in-band

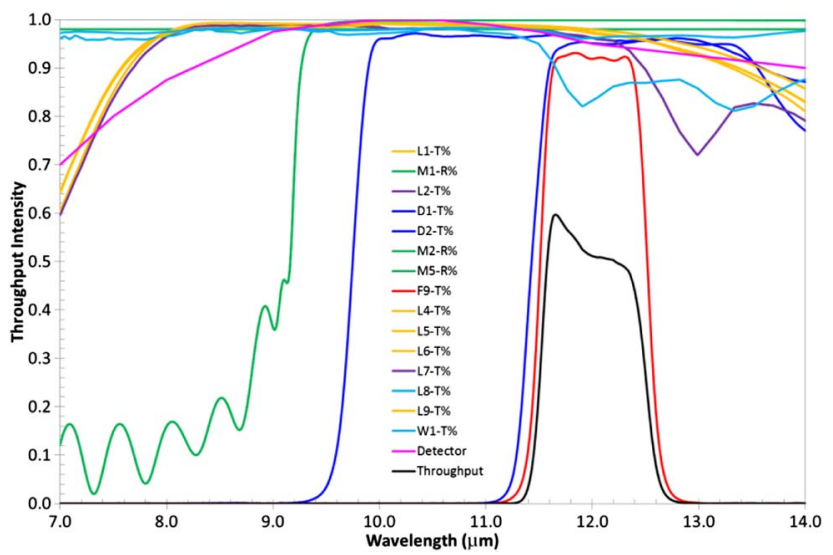

Fig. 18. Predicted total instrument throughput of Band 9 $(12.0 \mu \mathrm{m})$.

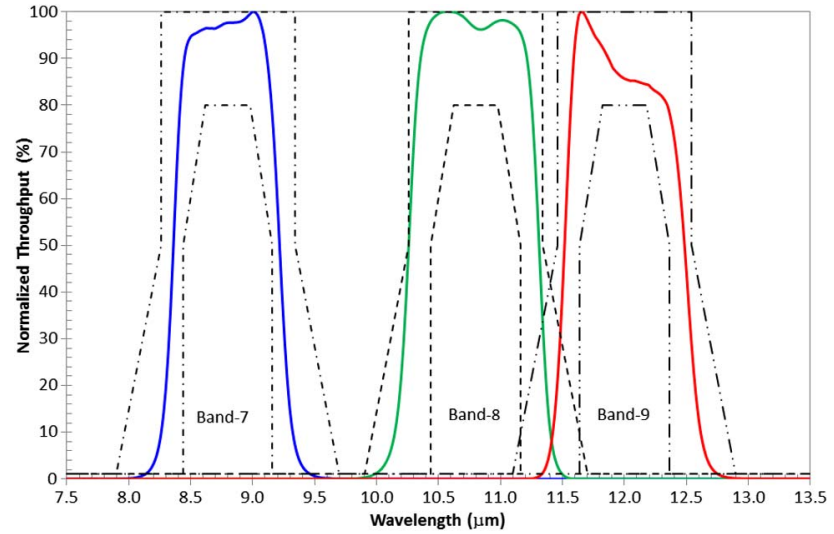

Fig. 19. Overlay of the predicted normalized wave band throughputs and their respective instrument templates.

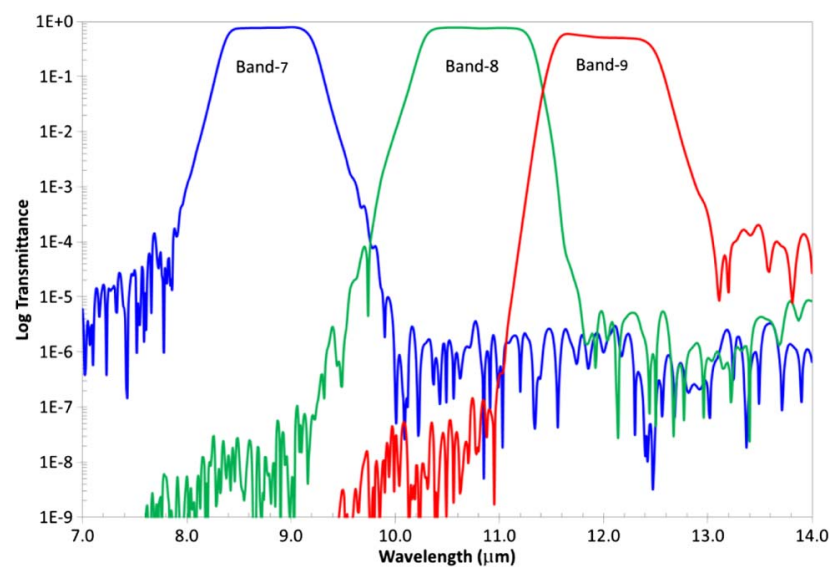

Fig. 20. Overlay of the predicted integrated system blocking performance.

system throughput for the MSI instrument channels is shown in Table 4.

The throughput $\bar{b}$ udget for each channel containing the contribution of each optical element is illustrated in Fig. 16 (Band 7 at $8.8 \mu \mathrm{m}$ ), Fig. 17 (Band 8 at $10.8 \mu \mathrm{m}$ ), and Fig. 18 (Band 9 at $12 \mu \mathrm{m}$ ). The throughput of the final system response is driven principally by the combinations of the dichroics and bandpass filters for the wave bands 7 and 8, and by additional contribution of high absorption from the germanium L8 lens across the Channel 9 wave band. The total MSI spectral responses in Fig. 19 show the correct positioning of all three channels compliant within the desired spectral template, together with the simulated integrated blocking intensity of $<10^{-4}$, as shown in Fig. 20 .

\section{Conclusions}

The design, manufacture, and testing of the MSI infrared coated optics has been described together with the choice of design, substrate, and coating materials. The development and successful fabrication of these coatings have required an adaptive range of coating techniques and procedures, with which the spectral challenges have been met. A spectral 
performance model for the thermal channels has been developed using simulation and measured spectral data. The use of the model has demonstrated that the predicted throughput response will comply with the specified channel requirements of the instrument.

This work has been supported under contract by Surrey Satellite Technology Limited (MS000163), UK, whose collaboration and support are gratefully acknowledged.

\section{References}

1. R. V. Gelsthorpe, A. Heliere, A. Lefebvre, J. Lemanczyk, E. Mateu, A. Perez-Albinana, and K. Wallace, "Aspects of the EarthCARE satellite and its payload," Proc. SPIE 7826, 78260G (2010).

2. B. Thies and J. Bendix, "Satellite based remote sensing of weather and climate: recent achievements and future perspectives," Met. Appl. 18, 262-295 (2011).

3. A. Pérez-Albiñana, R. Gelsthorpe, A. Lefebvre, M. Sauer, E. Weih, K.-W. Kruse, R. Münzenmayer, G. Baister, and M. Chang, "The multi-spectral imager on board the EarthCARE spacecraft," Proc. SPIE 7808, 780815 (2010).

4. A. Pérez-Albiñana, R. Gelsthorpe, A. Lefebvre, M. Sauer, K.-W. Kruse, R. Münzenmayer, G. Baister, M. Chang, J. Everett, A. Barnes, N. Bates, M. Price, M. Skipper, B. T. G. de Goeij, E. A. Meijer, F. van der Knaap, and A. Van't Hof, "The engineering model for the multispectral imager of the EarthCARE spacecraft," Proc. SPIE 8511, 85110L (2012).

5. L. G. Rojas, M. Chang, G. Baister, G. Hopkinson, M. Maher, M. Price, M. Skipper, T. Wood, and D. Woods, "The EarthCARE multispectral imager thermal infrared optical unit detection system design," Proc. SPIE 7826, 78260H (2010).

6. C. S. Evans, R. Hunneman, J. S. Seeley, and A. Whatley, "Filters for the $\nu_{2}$ band of $\mathrm{CO}_{2}$ : monitoring and control of layer deposition," Appl. Opt. 15, 2736-2745 (1976).

7. D. Woods, M. Chang, and G. Baister, "EarthCARE MSI TIR filter and dichroic coating specification," SSTL Ltd. reference document EC.RS.SSTL.MSI.00018, \#0119774 Version 5 (2010); otherwise unpublished.

8. D. Woods and J. Everett, "EarthCARE MSI TIR lens and mirror specification," SSTL Ltd. reference document EC.RS.SSTL.MSI.00036, \#0135617 Version 2 (2009); otherwise unpublished.
9. J. S. Seeley, H. M. Liddell, and T. C. Chen, "Extraction of Tschebyscheff design data for the lowpass dielectric multilayer," Opt. Acta 20, 641-661 (1973).

10. K. Matthews and K. Wright, Crystran Ltd. (private communication, 23 December 2008).

11. A. Smakula and M. Klein, "The plastic deformation of thallium halides in relation to crystal orientation," Report 1102, Project 8-23-02-002 (Engineer Research and Development Laboratories, Radiation Branch, 1949), submitted to U.S. Army.

12. W. Tropf and M. Thomas, "Infrared optical materials, inorganic optical materials," Proc. SPIE CR64, 137-169 (1996).

13. D. Blodgett, M. Thomas, D. Hahn, and S. Kaplan, "Longwave infrared absorption and scatter properties of $\mathrm{ZnS}$ and ZnSe," Proc. SPIE 5078, 137-147 (2003).

14. J. Pankove, Optical Processes in Semiconductors (Dover, 1971).

15. D. Khokhlov and M. O. Manasreh, Lead Chalcogenides: Physics and Applications, Vol. 18, Optoelectronic Properties of Semiconductors and Superlattices (Taylor \& Francis, 2003).

16. J. Seeley, R. Hunneman, and A. Whatley, "Infrared multilayer interference filter manufacture: supposed longwave limit," Appl. Opt. 18, 3368-3370 (1979).

17. K. Zhang, J. Seeley, R. Hunneman, and G. Hawkins, "Optical and semiconductor properties of lead telluride coatings," Proc. SPIE 1125, 45-52 (1989).

18. G. Hawkins, R. Hunneman, R. Sherwood, and B. Barrett, "Interference filters and coatings for mid-infrared astronomy (8-30 $\mu \mathrm{m})$," Proc. SPIE 4842, 43-55 (2002).

19. G. Hawkins, R. Sherwood, and K. Djotni, "Mid-infrared filters for astronomical and remote sensing instrumentation," Proc. SPIE 7101, 710114 (2008).

20. G. Hawkins, R. Hunneman, R. Sherwood, and B. Barrett, "Infrared filters and coatings for the high resolution dynamics limb sounder," Appl. Opt. 39, 5221-5230 (2000).

21. J. Ruffner, M. Himel, V. Mizrahi, G. Stegeman, and U. Gibson, "Effects of low substrate temperature and ion assisted deposition on composition, optical properties, and stress of ZnS thin films," Appl. Opt. 28, 5209-5214 (1989).

22. R. Hunneman, R. Sherwood, C. Deeley, and R. Spragg, "Achieving accurate FTIR measurements on high performance bandpass filters," in Eleventh International Conference on Fourier Transform Spectroscopy (American Institute of Physics, 1997), pp. 435-438.

23. G. Hawkins and R. Sherwood, "Cooled infrared filters and dichroics for the James Webb Space Telescope mid-infrared instrument," Appl. Opt. 47, C25-C34 (2008).

24. G. Hawkins and R. Hunneman, "A spectral performance model for the High Resolution Dynamics Limb Sounder (6-18 $\mu \mathrm{m})$," Infrared Phys. Technol. 41, 239-246 (2000). 